\title{
Patient-focused HTAs
}

HTA can be described as a research-based, practice-oriented assessment of relevant evidence and knowledge on the direct and intended effects of healthcare technologies, as well as the indirect and unintended consequences (4). It is also viewed as a multidisciplinary field of policy analysis that studies the medical, ethical, social, and economic implications of the development, diffusion, and use of a health technology (5).

Patients have a unique understanding of what it's like to live with a medical condition, with experience of using health services and health technologies that should provide "relevant knowledge" about the intended and unintended effects of using technologies. Also, given the policy imperative for patient-focused health care and informed patient choice, for political legitimacy patients should surely be involved in the "multidisciplinary" process of HTA (2). However, HTA is not only a policy analysis, but also a scientific process. Evidence must be critically scrutinized by researchers and knowledge balanced against other inputs in the deliberative decision-making process.

In the past, patients' views have often been considered anecdotal or biased and their views have not been included in HTA. To ensure that patients' knowledge and experience are used in HTA, good quality evidence about patient aspects is needed alongside an HTA process that supports effective patient involvement. The challenge is to demonstrate to HTA doers the value and quality of humanistic and social studies as evidence. Meanwhile, theories on participation and partnership are important for effective involvement (1). This Theme Section on Patients in HTA presents eleven papers that review work on patient evidence and involvement in HTA and propose new methods to create patient-focused HTAs.

Tjørnhøj-Thomsen and Hansen review the knowledge generation process in HTA. They critically examine the range of decisions made throughout the process and show how these are affected by the stance of the researchers and contributors. They stress the need to be more explicit about knowledge production in HTA to ensure that patients' knowledge are included fairly.

Hansen et al have produced a fascinating review of fiftyeight Danish HTAs spanning the last decade. This shows an impressive record. All reports included patient aspects, using systematic reviews, primary research, or both, and the range of methods used and impact on final conclusions is documented. This provides an excellent example for other HTA Agencies about how to gather patient evidence and the impact it had.

Moran and Davidson and Ahern et al. have undertaken reviews of public involvement in their HTA organizations. Common themes emerge such as greater involvement in particular stages of the HTA process and the need for support of individual public contributors, alongside organizational commitment to public involvement. There are differences too. In the United Kingdom, there is little input to publication and dissemination. In Australia, the importance of clear information to patients is recognized and patients are involved in that information production.

Recognizing the importance of information for patients, Bastian et al. have undertaken research to determine what aspects of conclusions from systematic reviews are of interest to patients and developed a framework for information priority setting. They determine that the best evidence to communicate to patients is right, relevant and interesting. Pasternack et al. and Izquierdo et al. provide practical examples of how to develop patient decision aids from HTAs in relation to breast cancer. Both approaches are systematic and thorough, but the lack of patient involvement in the early stages of development is noted as a weakness.

There is no one-size-fits-all methodological solution to the elicitation of patients' needs, preferences and experiences that will produce robust evidence. The key research questions relating to patient aspects are often hard to define and must be determined in an iterative process. Danner et al. present an 
Analytic Hierarchy Process that uses iterative pairwise comparisons of endpoints to determine the most important. In a study of antidepressants, patients and health professionals chose the same six endpoints, but patients were more interested in shorter term outcomes. Street et al. show how to use the latest information sources from the web to understand community views about the emotive issue of disinvesting services for assistive reproductive technology. Their study describes how these sources can be reviewed systematically and the results show divergent views, providing important socio-political aspects. Ring et al. help us demystify synthesis of qualitative evidence. They present eight key methods and show how they have been used in HTAs and their potential for use in the future.

We can promote the necessity of including evidence about patient aspects in HTA and involving patients in the HTA process, but we need to demonstrate the impact. Staniszewska et al. give us a helpful start by drawing on two systematic reviews that identify weaknesses in studies that report patient involvement. As a result, they develop the GRIPP checklist to improve the conduct and reporting of patient involvement in health research. More work needs to be done to demonstrate the impact of patient involvement in HTA and the HTAi Interest Group on Patient/Citizen Involvement is working on this.

Finally, we are grateful to a range of stakeholders for considering the question posed in a letter to the Journal about whether a health professional can represent patient views. This suite of letters is mandatory reading for any HTA doer who wants to involve patients. It presents the arguments for and against and we think the answer is, "it depends." We believe that robust patient evidence about a range of patient aspects is needed and that patients should be involved throughout the HTA process from scoping of relevant questions and endpoints, through to contribution of robust evidence, appraisal of technology consequences and communication of HTA information to patients. The manner of that involvement will depend on the aim and stage of the HTA (3). Furthermore, we must remember that patients are not a homogeneous group: they differ in relation to gender, age, education, socio-economic status, etc., and have a variety of needs, experiences, and beliefs. We must obtain their views and knowledge about the intended and unintended consequences of using a health technology to ensure that we generate patient-focused HTAs.

Karen M. Facey, BSc, PhD

Chair HTAi Interest Group on Patient/Citizen Involvement in

HTA

Woodlands Lodge

Buchanan Castle Estate

Drymen G63 OHX, UK

Email: k.facey@btinternet.com

Helle Ploug Hansen, MA (extended Master), PhD

Professor in Humanistic Rehabilitation Research

Institute of Public Health

Research Unit; Health, Man \& Society and National

Research Centre for Cancer Rehabilitation

University of Southern Denmark

J.B. Winslфws Vej 9

DK-5000 Odense C, Denmark

Email: hphansen@health.sdu.dk

\section{REFERENCES}

1. de Wit MP, Berlo SE, Aanerud GJ, et al. European League Against Rheumatism recommendations for the inclusion of patient representatives in scientific projects. Ann Rheum Dis. 2011;70:722-726.

2. Facey K, Boivin A, Gracia J, et al. Patients' perspectives in HTA: A route to robust evidence and fair deliberation. Int $J$ Technol Assess Health Care. 2010;26:334-340.

3. Gauvin F-P, Abelson J, Giacomini R, et al. "It all depends": Conceptualizing public involvement in the context of health technology assessment agencies. Soc Sci Med. 2010;70:15181526.

4. Health Technology Assessment International. HTAi strategic plan 2009 - 2013 (section on background and environment). Canada: HTAi; 2009 www.htai.org/fileadmin/HTAi_Files/ StrategicPlan2009To2013Materials/HTAi2009To2013Strategic Plan.pdf (accessed July 4, 2011).

5. International Network of Agencies for Health Technology Assessment. HTA resources - definition of technology assessment. Sweden: INAHTA. www.inahta.org/HTA/ (accessed July 4, 2011). 